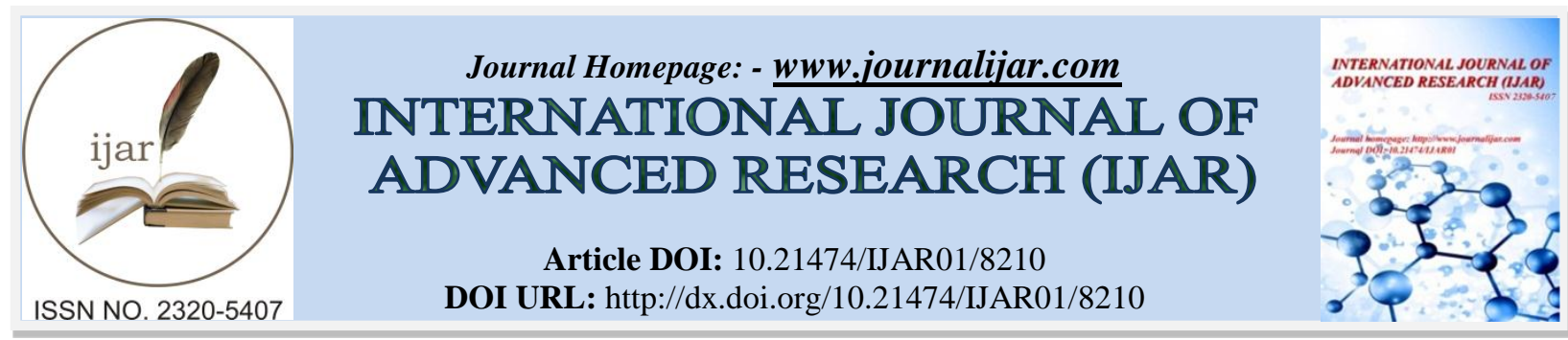

RESEARCH ARTICLE

\title{
CAN DIODE LASER SUPPORT REMINERALIZATION POTENTIAL OF CPP-ACPF ON DEMINERALIZED ENAMEL?.
}

\author{
Rehame M. Ameen ${ }^{1}$, Rania A. Awwad ${ }^{2}$ and Asia M. Elsheflak. \\ 1. Professor of Oral Biology, Faculty of Dentistry, Ain Shams University. \\ 2. Lecturer of Oral Biology, Faculty of Dentistry, Ain Shams University.
}

\section{Manuscript Info}

Manuscript History

Received: 16 October 2018

Final Accepted: 18 November 2018

Published: December 2018

\begin{abstract}
Recent studies recognized that incorporation of fluoride into the Casein Phospho-Peptide - Amorphous Calcium Phosphate (CPP-ACP) structure forming (CPP-ACPF): (GC MI paste Plus), produced greater remineralization than the CPP-ACP alone. The aim of the present study was to assess the efficacy of diode laser irradiation on remineralization potential of CPP-ACPF cream on induced human surface enamel demineralization, using Scanning Electron MicroscopeEnergy Dispersive X- ray analysis SEM-EDAX analyser.

Materials \& Methods: forty intact premolars were collected and thirty of them were immersed in a demineralizing solution for 10 weeks to induce caries like lesions and then were divided into four groups, each consist of 10 teeth. In C-ve group the specimens were immersed in normal saline only $\mathrm{C}+\mathrm{ve}$ group specimens were subjected only to demineralizing solution. In Group (CPP-ACPF alone) this group was treated with application of a sufficient thickness of CPP-ACPF cream for 4 minutes. In group (CPP-ACPF plus $970 \mathrm{~nm}$ diode laser), the demineralized enamel was covered with CPP-ACPF cream for 4 minutes and $(970 \mathrm{~nm}, 1 \mathrm{mw}$ diode laser) was employed at the last one minute of cream application with distance of $5 \mathrm{~mm}$ from the tooth surface.

Results: Although the highest mean total weight percentages of $\mathrm{C}, \mathrm{Ca}$, $\mathrm{P}, \mathrm{F} \%$ wt and $\mathrm{Ca} / \mathrm{P}$ ratio resulting from the EDAX analysis of enamel surface was observed in group (CPP-ACPF plus 970nm diode laser). However, ANOVA displayed no significant differences was found between the $\mathrm{C}+\mathrm{ve}$ and experimental groups ( $\mathrm{p}>0.05$ ).

Conclusion: The exposure to the demineralizing agent significantly decreased the calcium, phosphorous and fluoride level of enamel surface and altered enamel surface morphology. When demineralized enamel was followed by CPP-ACPF alone a better morphological result as well as increased ionic wt $\%$ was observed compared to $\mathrm{C}+\mathrm{v}$ group.CPP-ACPF plus 970nm laser application resulted in more reduction in morphological enamel defects compared to $\mathrm{C}+\mathrm{ve}$ group.
\end{abstract}

Copy Right, IJAR, 2018,. All rights reserved.

Corresponding Author: - Rania A. Awwad. 


\section{Introduction:-}

Demineralization and remineralization have important role on the hardness and strength of tooth enamel. The battle to keep teeth strong and healthy is dependent upon the ratio between demineralization and remineralization (Margeas, 2006). Chemical demineralization of teeth is caused by acidic attack through two primary means: dietary acid consumed through food or drink and microbial attack from bacteria present in the mouth (Scaramucci et al, 2015).

Remineralization is the natural repair and production process of restoring minerals again in the form of mineral ions to the hydroxyapatite's lattice. Below critical $\mathrm{pH}$, demineralization occurs while above critical $\mathrm{pH}$, re-mineralization occurs (Moslemi et al,2009). Casein phosphopeptide-amorphous calcium phosphate (CPP-ACP is a milk-derived product that strengthens and remineralizes the demineralized enamel white lesion and helps in prevention of dental caries (Hemagaran, et al, 2013). Recent studies recognized that incorporation of fluoride into the CPP-ACP structure (CPP-ACPF) (GC MI paste Plus) produced greater remineralization than the CPP-ACP alone (Patil, et al, 2013). In comparison to other lasers, diode laser is more economical. Unfortunately, diode lasers are only used in soft tissue but not practical for the removal of hard tissues. In soft tissue treatment diode laser have similar effect to the Neodymium Yttrium Garnet Laser (Nd: YAG) which is used for hard tissues and not far away wave lengths. Diode laser effect on demineralized enamel surfaces coupled with the use of the re-mineralizing agent application are still unclear. So there is a definite need for more laser comparative studies to compare their performance clinically to determine which system can be used for short time and less cost. However, there are few studies regarding the effectiveness of this agent combined with low power diode laser on demineralized white lesion (Geraldo-Martins et al, 2013). Thus, the aim of this study was to investigate the efficacy of 970nm Diode laser irradiation on remineralization potential of CPP-ACPF cream on human surface enamel induced caries like lesions, using Scanning Electron Microscope- Energy Dispersive X- ray analysis SEM-EDAX.

\section{Materials and Methods:-}

\section{Teeth:}

Forty non-carious freshly extracted human premolars - extracted for orthodontic reasons- were collected from dental clinics, cleaned and stored in normal saline solution at room temperature. Teeth were examined visually by a magnifying lens and the specimens with crack or defects in enamel were discarded and replaced.

\section{Materials :}

1. Demineralizing agents: $2.2 \mathrm{mM} \mathrm{CaCl} 2,2.2 \mathrm{mM} \mathrm{NaH} 2 \mathrm{PO} 4$, solution and $50 \mathrm{mM}$ acetic acid prepared at oral biology lab of Ain Shams University.

2. CPP-ACPF cream (Recaldent) from GCMI Past Plus Japan company.

3. $970 \mathrm{~nm}$ Diode laser system (SIRO-laser advanced; Sirona Dental Systems Gmbh, Benheim, Germany) with the following technical features: continuous modulated mode radiation power is 1-1000 mW with wave length 970 $\mathrm{nm}$, and the exposure time ranges from 1-99 sec., at National Research Centre.

4. Scanning Electron microscope and Energy Dispersive X-ray analysis (SEM-EDAX analyser) with S-UTW detector (EDAX INC, Mahwah, N j, USA) at Mansoura University.

All teeth were coated with two consecutive layers of acid resistant nail varnish except for a $3 \times 3 \mathrm{~mm}$ window on the mid of the buccal surface, which served as the treatment area. Teeth were examined by SEM-EDAX analyser, any teeth showed

enamel defects or extreme EDAX analyses findings was discarded and replaced. After examination, specimens were immersed in a demineralizing solution after checking it is PH (4.8) for 10 weeks to induce caries like lesions (Shahabi et al, 2013). Then teeth were randomly divided as follows into a control group and two experimental groups where each group include10 teeth.

Table 1:-showing Distribution of the study groups.

\begin{tabular}{|l|l|l|}
\hline Experimental groups & Application & Number of teeth \\
\hline Control negative group & Normal saline & 10 \\
\hline Control positive group & Demineralizing agent & 10 \\
\hline
\end{tabular}




\begin{tabular}{|l|l|l|}
\hline APP-ACPF alone group & CPP-ACPF cream & 10 \\
\hline CPP-ACPF plus 970nm laser group & $\begin{array}{l}\text { CPP-ACPF cream plus 970nm } \\
\text { laser }\end{array}$ & 10 \\
\hline
\end{tabular}

\section{Samples examination}

Each tooth in both control and experimental groups was then prepared for examination by washing thoroughly under running water for $30 \mathrm{sec}$., left to dry in open air. Each tooth was covered with special cap made of a carbon meshwork $\left(1 \mathrm{~mm}^{2}\right)$. All the meshwork was covered with clay except the middle one third of the labial surface. Then the teeth were mounted on the SEM holder using removable adhesive and specified a certain area on a certain square of the meshwork to be examined with SEM under the magnification of (2000). Surface c, ca, p and $\mathrm{f}$. wt\% were measured by EDAX analyser.

\section{Statistical analysis:-}

1. Results of the present study were collected, tabulated, then subjected to statistical analysis to interpret the significant difference between various ions wt $\%$ of $\mathrm{C}, \mathrm{Ca}, \mathrm{P} \mathrm{CalP}$ and $\mathrm{CalF}$ ratio of enamel surface in all groups.

2. Statistical analysis was performed using T-test and ANOVA for comparison between Mean and SD of five groups.

\section{Results:-}

Scanning Electron Microscope finding:

\section{Control group}

Scanning Electron Microscope examination of the specimens of control group shown enamel surface which were mostly represented as crusted structurless areas masking enamel surface. Irregular different sized pits and pores were noticed. Wide crater like depression, few microcracks and fish scale like appearance were also observed. (Fig.1)

\section{CPP-ACPF alone group:}

Scanning Electron Microscope examination of enamel surface of CPP-ACPF alone group represented numerous different sized granular particles with fine porosities. While in few limited areas the enamel surface represented thin structurless enamel crusts masking different sizes of wide craters like depression with ill-defined irregular perikymata grooves were seen (Fig 2).

\section{CPP-ACPF plus 970nm laser:}

Examination by SEM of middle third of buccal surface, in this group represented more regular and smooth enamel surface with few fine granular to globular particles deposits. Confined areas of smooth glazed molten enamel were also observed. Enamel rod ends were hardly defined, together with undefined perikymata (fig 3).

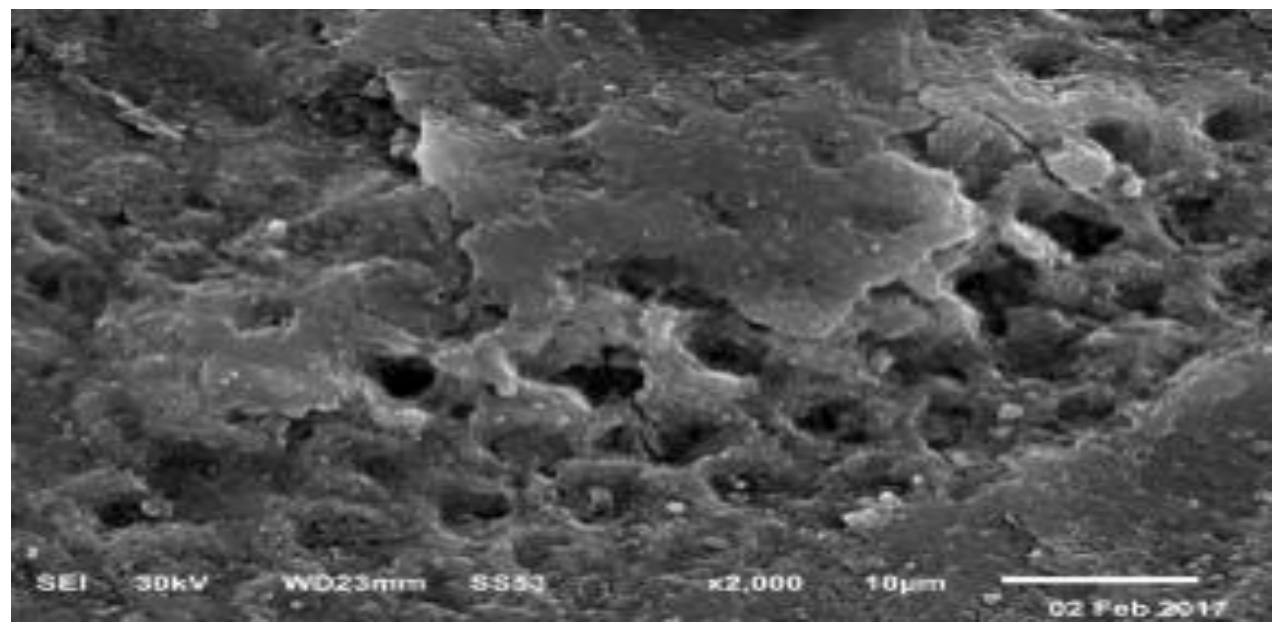

Figure 1:-SEM image of enamel surface of the control group. 


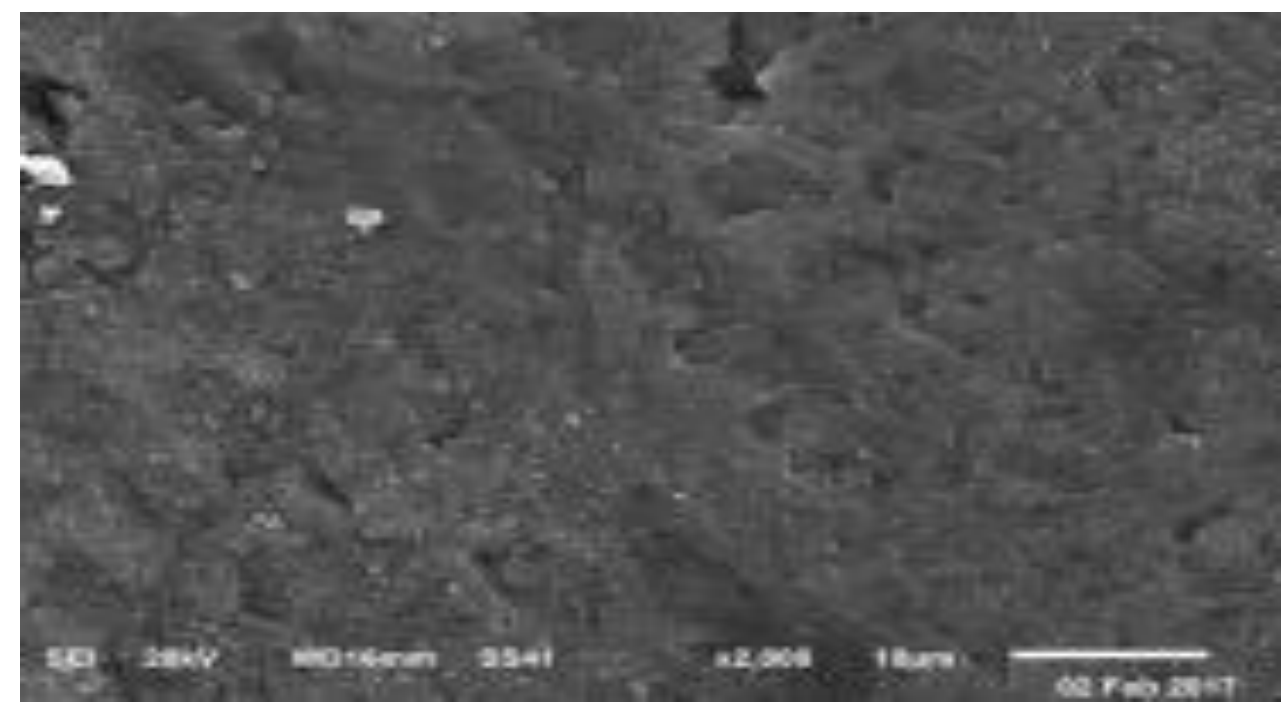

Figure 2:-SEM image of the enamel surface of group 2 (treated with CPP-ACP alone).

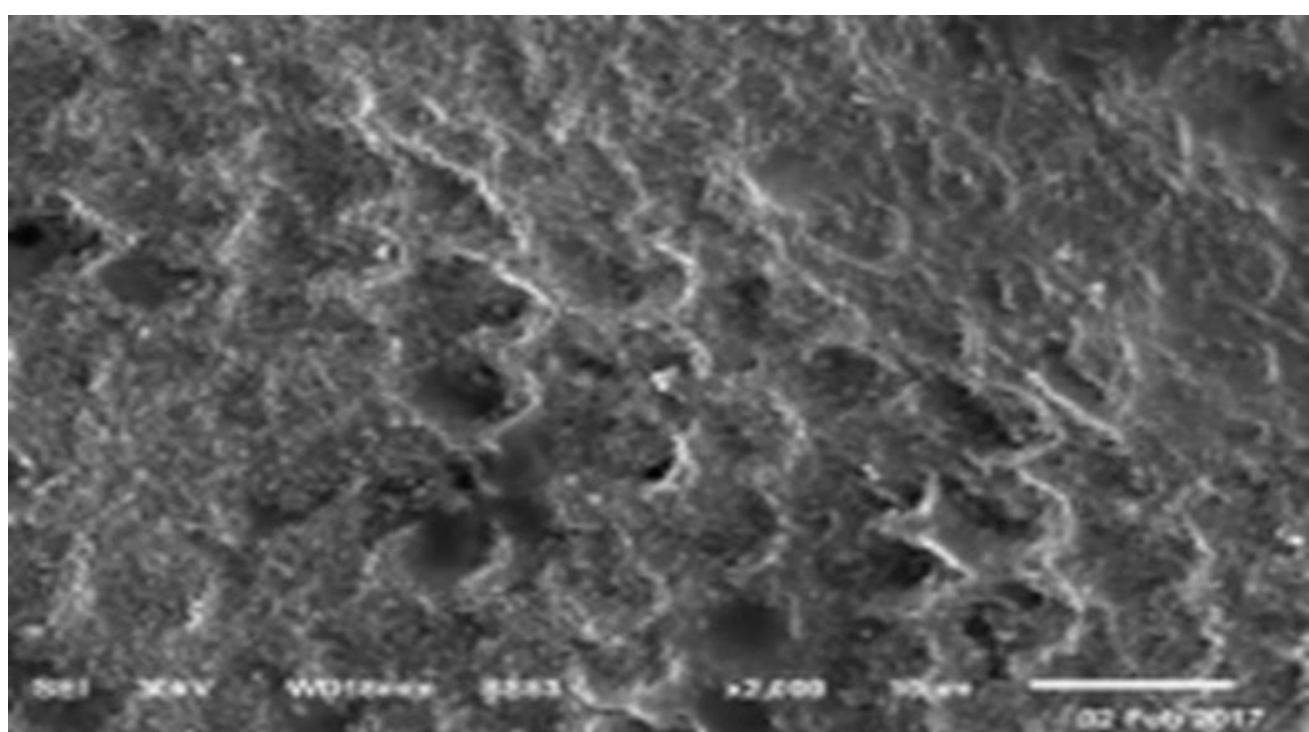

Figure 3:-showing EMS image of enamel surfaces of group 3( treated with CPP-ACPF cream and 970nm 500 mwatt).

\section{Statistical results:}

The mean surface $\mathrm{Ca}, \mathrm{P}, \mathrm{F}$ wt $\%$ in group 2 was higher than in control group. Also, the mean surface $\mathrm{C}, \mathrm{Ca}, \mathrm{P}, \mathrm{F}$ wt $\%$ in group3 was higher than in Control group. These differences were statistically significant.

Although the highest mean total weight percentages of $\mathrm{C}, \mathrm{Ca}, \mathrm{P}, \mathrm{F} \%$ wt and $\mathrm{Ca} / \mathrm{P}$ ratio resulting from the EDAX analysis of enamel surface was observed in group ( CPP-ACPF $970 \mathrm{~nm}$ diode laser) and the lowest one and control +groups. However, ANOVA displayed no significant differences was found between the C+ve and experimental groups ( $>0.05)$. 


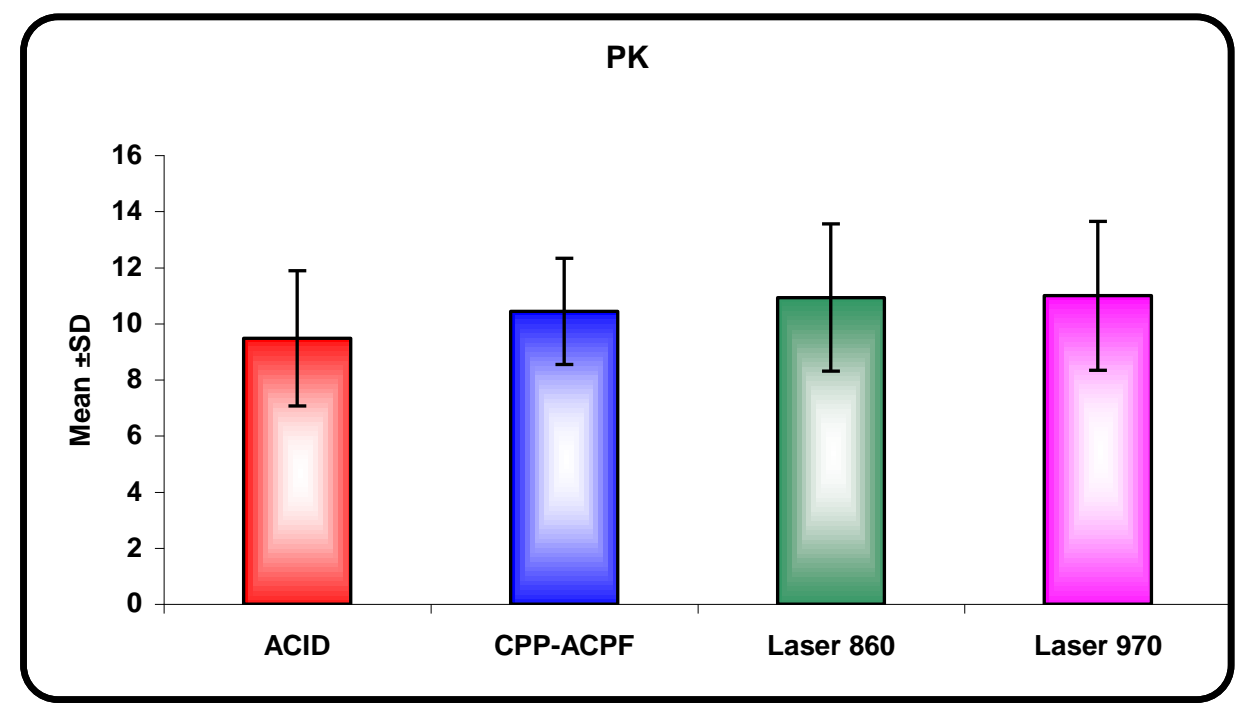

Figure 4:-Bar chart representing mean weight $\%$ and SD of the phosphorus of 3 groups.

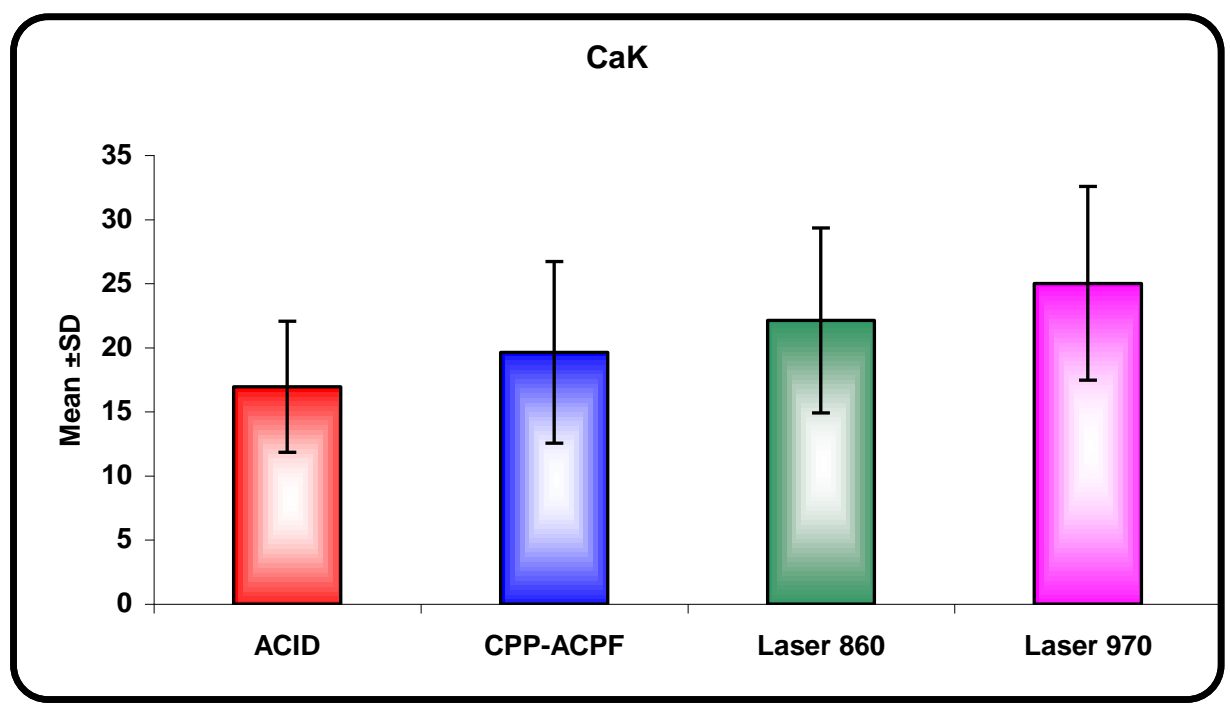

Figure 5:-Bar chart representing mean weight $\%$ and SD of the calcium in all 3 groups. 


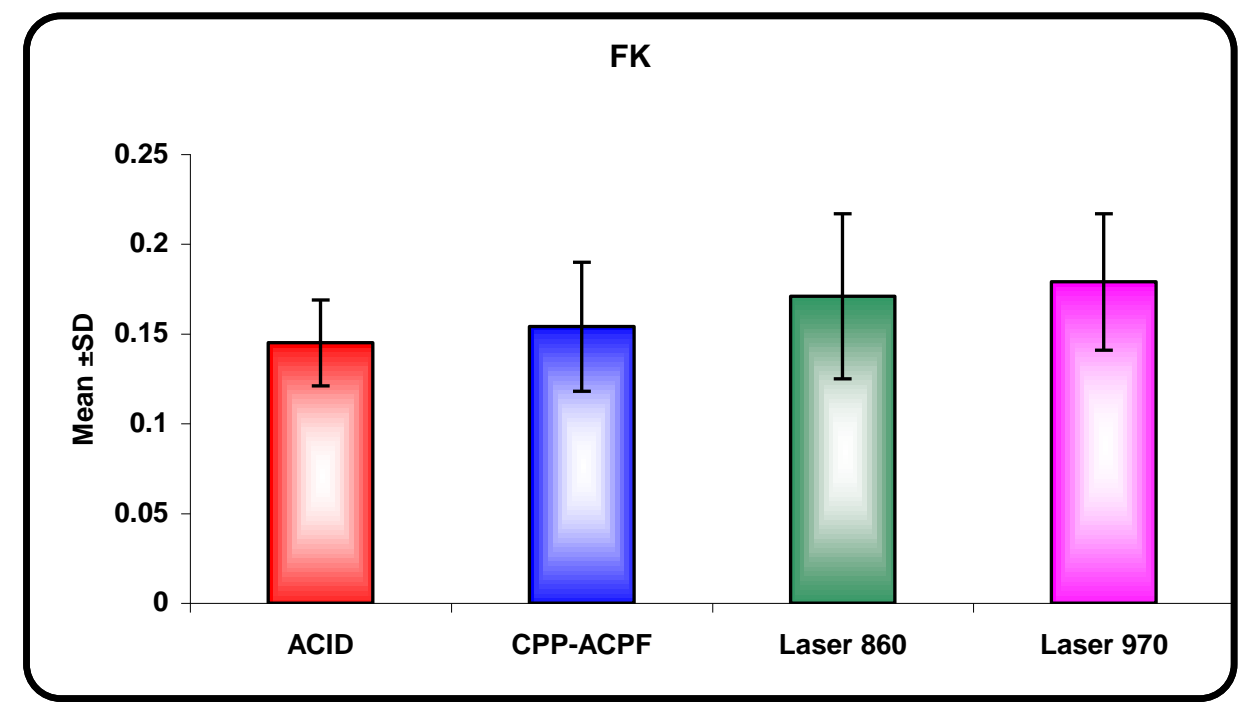

Figure 6:-Bar chart representing mean weight $\%$ and SD of the fluoride in all 3 groups.

\begin{tabular}{|c|c|c|c|c|c|c|c|c|}
\hline \multirow[t]{2}{*}{ Groups } & \multicolumn{6}{|l|}{ PK } & \multicolumn{2}{|c|}{ ANOVA } \\
\hline & \multicolumn{3}{|c|}{ Range } & Mean & \pm & SD & \multirow{4}{*}{$\begin{array}{l}\mathrm{F} \\
0.843\end{array}$} & \multirow{4}{*}{$\begin{array}{l}\text { P-value } \\
0.479\end{array}$} \\
\hline ACID & 5.61 & - & 13.23 & 9.479 & \pm & 2.404 & & \\
\hline CPP-ACPF & 6.76 & - & 13.08 & 10.442 & \pm & 1.895 & & \\
\hline Laser 970 & 5.81 & - & 14.59 & 10.998 & \pm & 2.656 & & \\
\hline
\end{tabular}

Table 1:-descriptive statistics, result, and Anova test for the comparison between mean and SD of the phosphorus of 3 groups.

\begin{tabular}{|c|c|c|c|c|c|c|c|c|}
\hline \multirow[t]{2}{*}{ Groups } & \multicolumn{6}{|l|}{$\mathrm{CaK}$} & \multicolumn{2}{|c|}{ ANOVA } \\
\hline & \multicolumn{3}{|l|}{ Range } & Mean & \pm & SD & \multirow{4}{*}{$\begin{array}{l}\mathrm{F} \\
2.558\end{array}$} & \multirow{4}{*}{$\begin{array}{l}\text { P-value } \\
0.070\end{array}$} \\
\hline ACID & 10.13 & - & 26.21 & 16.951 & \pm & 5.112 & & \\
\hline CPP-ACPF & 10.85 & - & 32.53 & 19.645 & \pm & 7.083 & & \\
\hline Laser 970 & 11.12 & - & 37.74 & 25.012 & \pm & 7.554 & & \\
\hline
\end{tabular}

Table2:-descriptive statistics, results, and Anova test for the comparison between mean and SD of the calcium of 3 groups.

\begin{tabular}{|c|c|c|c|c|c|c|c|c|}
\hline \multirow[t]{2}{*}{ Groups } & \multicolumn{6}{|l|}{ FK } & \multicolumn{2}{|c|}{ ANOVA } \\
\hline & \multicolumn{3}{|c|}{ Range } & Mean & \pm & SD & \multirow{4}{*}{$\frac{\mathrm{F}}{1.775}$} & P-value \\
\hline ACID & \begin{tabular}{|l|}
0.11 \\
\end{tabular} & - & 0.18 & 0.145 & \pm & 0.024 & & \multirow[t]{3}{*}{0.169} \\
\hline CPP-ACPF & \begin{tabular}{|l|}
0.11 \\
\end{tabular} & - & 0.21 & 0.154 & \pm & 0.036 & & \\
\hline Laser 970 & \begin{tabular}{|l|}
0.12 \\
\end{tabular} & - & 0.24 & 0.179 & \pm & 0.038 & & \\
\hline
\end{tabular}

Table3:-descriptive statistics, results, and Anova test for the comparison between mean and SD of the fluoride of 3 groups.

\section{Discussion:-}

The CPP-ACPF treatment had previously shown to enhance the resistance of enamel by increasing the uptake of Ca, $\mathrm{P}, \mathrm{F}$ by enamel and consequently reducing the acid dissolution rate of enamel (Jayarajan et al., 2011). Thus this study aimed to reconstructing this model using CPP-ACPF cream with diode laser.

Freshly extracted sound human premolar teeth were chosen to be samples of this study and collected from orthodontic clinics, which make the study more realistic, predicting the possible effects of CPP-ACPF with diode laser on sound enamel surface. The middle one third of the buccal surface was used in the present study as monitoring area because it contains a moderate percentage of the elements between cuspal and cervical enamel. This was based on a research carried by (Gutiérrez-Salazar et al., 2003) who studied the difference between the elemental composition of the cuspal and cervical enamel of the human teeth and their results revealed that the calcification was lower at cervical enamel 
than that of the cuspal areas as the level of $\mathrm{Ca}$ and $\mathrm{P}$ wt $\%$ was less in the cervical areas. Also, the overall prevalence of enamel white lesion related to fixed orthodontic appliance treatment found at the area of the bracket sites, where the food was impacted ranged from (2-96\%) (Ogaard et al., 2008).

In this study, the induction of caries like lesion was done by demineralizing solution consisting of $2.2 \mathrm{mM} \mathrm{CaCl} 2$, $2.2 \mathrm{mM}, \mathrm{NaH} 2 \mathrm{PO} 4$, and $50 \mathrm{mM}$ acetic acid (ph4.8) for 10 weeks. This solution was used perfectly as acidic media by many previous studies for demineralization of enamel surface as a model of enamel caries like lesion (Shahabi et al., 2012; Bassett et al., 2013).

The CPP-ACPF cream was used in current study because, the incorporation of $0.2 \%$ or $900 \mathrm{ppm}$ of NaF into CPPACP (Tooth Mousse Plus ${ }^{\mathrm{TM}}$ ) was produced as CPP-ACPF (Tooth Mousse Plus ${ }^{\mathrm{TM}}$ ) which had more benefit to localize calcium, phosphate, and fluoride at the tooth surface than the CPP-ACP. (CPP-ACP) cream was preferred as it is a milk-derived product. The CPP part of this complex is used to localize amorphous calcium phosphate (ACP) in dental plaque and provide a state of supersaturation with respect to enamel. In addition, amorphous calcium fluoride phosphate (ACFP) forms because of interaction between the CPP-ACP and fluoride ions, and stabilizes by the CPP on the tooth surface (Rose, 2000). The warning was reported against treating visible demineralizing like lesion with concentrated fluoride agents immediately, because this procedure will arrest the lesions and prevent complete repair by surface hyper-mineralization. Hence, the use of fluoride alone as a re-mineralizing agent in clinical situation is not beneficial. With the use of low fluoride concentration as is present in CPP-ACPF $(0.2 \%$ or $900 \mathrm{ppm}$ of $\mathrm{NaF})$, there is a complex localization of free calcium phosphate and fluoride ion activities, which helps in maintaining a state of supersaturation by suppressing demineralization. Thus CPP-ACPF (Tooth Mousse Plus ${ }^{\mathrm{TM}}$ ) is an excellent local slow-delivery system to treat the white spot lesion (Holler et al 2002). So, this study aimed to increase the calcium phosphate and fluoride ions even with no much increase of fluoride wt $\%$ on this lesion.

Also, hydroxyapatite is the principal component of human teeth and its crystallinity is one of the resistant factors against acid agents. The use of EDAX permits evaluating the quantitative changes by wt $\%$ in the mineral composition of surface enamel in localized cubic microns areas that have undergone to laser treatment, this is because any change can be assumed as hydroxyapatite variation, consequently the enamel's degree of crystallization and correlates the distribution of various elements to histological structure of the enamel (Samuel and Rubinstein, 2001).

using of diode laser in this study was indicated because it is a relatively inexpensive when compared to high power lasers and has similar effects in soft tissue to the Nd: YAG laser, which is used for hard tissue and it is wave length $(1064 \mathrm{~nm})$ not far away to the wave length of diode laser (970nm) (deSant'anna et al., 2009). So, its application for prevention or remineralization of demineralizing lesion in short time with maximum effects seems interesting. Also, the efficacy of CPP-ACPF for promoting enamel remineralization has not been sufficiently investigated. Therefore, there was still a need to search more about its beneficial effects.

The diode laser compared to high power laser considered safer regarding to their effects on enamel surface morphology, had less hazard effect on the tooth structure, less cracks, less rougher enamel surface, which can make the tooth more susceptible to adhesion to bacterial plaque and acid, and less liability of pulp necrosis occurring where it is temperature not rise more than 50C (Herviet et al. 2014; Ahrari et al., 2014).

In some studies, authors as that of ( $\mathrm{Li}$ et al., 2007) proposed that application of a photo-absorbing cream plus low power laser on the enamel surface, which should be proportional to the wavelength of the low power laser, can efficiently intensify energy of the absorption and transform it to heat, thus providing in photochemical changes in the enamel surface and photo-thermal effects which responsible for the modification in dental hard tissue structure including reduction of carbonate content and alterations in organic matters which lead to increase in the acid resistance so in this study it was tried to enhance these properties by using low power diode laser.

In this study, SEM examination result of control positive group revealed a relatively structure-less crusted area masking enamel surface interrupted with irregular depression, porosities and irregular different sized pits. Few micro-cracks and crater like depression were also observed with globular deposits. This coincident with (Patil et al., 2013) who evaluated the re-mineralizing potential of CPP-ACPF on artificially demineralized human enamel and they reported that, all SEM microphotographs taken after $48 \mathrm{~h}$ of demineralization showed irregular surface with porosities and loss of some surface enamel. This study also in agreement with (Jayarajan et al., 2011) who found 
that, the SEM configuration of demineralized enamel micrographs was apparent with certain porous defects and irregularities.

In current study, SEM examination results of CPP-ACPF alone revealed numerous different sized granular particles, amorphous crystals were concentrated along the porous defects on the enamel surface with fine porosities and few depressions which were seen in some areas. These results agreed with (Asl-Aminabadi et al., 2015) who studied the laser-casein phospho-peptide effect on remineralization of early enamel lesions in primary teeth and their results revealed that numerous granular particles and amorphous homogeneous crystals were seen on the enamel surface. This also came in accordance with (Jayarajan et al., 2011) who reported that the areas of calcified deposits were more evident and seen concentrated along the porous defects on the enamel surface.

In current study, SEM examination results of CPP-ACPF plus $(970 \mathrm{~nm})$ diode laser revealed a relatively smooth more homogeneous structure-less enamel surface compared to those in the groups treated with CPP-ACP alone. Fewer cracks were observed on the glazed enamel surface consistent with the numerous occurrences of granular and small crystalline deposits on enamel surface with less surface depression and micro-porosities. Poorly defined perikymata, crater like depressions were also seen. These results were explained by (de Sant'anna et al., 2009) who believed that the decrease in enamel solubility after laser treatments is due to changes in the infrastructure, such as water and carbonate content reduction, increase in hydroxyl ions, pyrophosphates formation, and protein decomposition. Another possibility would be to change and possibly destroy the organic material found in the interprismatic space and create such microspaces that can act as sites of deposition of ions and formation of granular, globular particles, amorphous homogenous crystals arranged on the enamel surface. Also, these findings can be further explained by (Asl-Aminabadi et al., 2015), who believed that, the photo-thermal melting and re-solidifying processes caused by low or high powered laser treatment, can reduce enamel permeability, cause fusion of enamel surface and formation of glazed surface. While the CPP-ACPF increases the subsurface concentration of calcium, phosphate and as a result, the hydroxyapatite and fluorapatite crystals formation will occur on the enamel surface so the surface became more homogeneous surface.

Statistical analysis of $\mathrm{C}+\mathrm{ve}$ group showed that, after immersion in demineralizing solution, there was a significant increase in $\mathrm{C}$ wt\% according to EDAX results correlated to significant decrease in $\mathrm{Ca}, \mathrm{P}, \mathrm{F}, \mathrm{Ca} \mathrm{P}, \mathrm{CalF}$ content which was also reflected on SEM results documenting the changes on enamel surface morphology. As known that the carbonate may substitute $\mathrm{OH}$ or $\mathrm{P}$ in apatite lattice so increase $\mathrm{C}$ content reflects the decrease in enamel hardness with significant decrease of $\mathrm{Ca}$ content, due to the release of $\mathrm{Ca}$ from the enamel surface as a result of surface hydroxyapatite destruction caused by the demineralizing solution (Xu et al., 2012).

In this study, after application of CPP-ACPF alone, there was a significant decrease in C content with increase in $\mathrm{Ca}, \mathrm{P}, \mathrm{F}$, and $\mathrm{CalP}$, ratio compared to $\mathrm{C}+\mathrm{ve}$ group with no significant increase in $\mathrm{Ca} / \mathrm{F}$. However, this reflected an increase in the enamel remineralization and decrease in its solubility which was confirmed by SEM results. These results were in agreement with (Jayarajan et al., 2011) who studied the efficacy of CPP-ACP and CPP-ACPF on enamel remineralization using scanning electron microscope. In this study some of the specimens were rubbed with CPP-ACP paste and the others were rubbed with CPP-ACPF paste for 4 minutes. The result showed valuable statistically significant amount of remineralization of CPP-ACPF group on demineralized enamel surface compared to CPP-ACP cream group.

In this study, after application of CCP-ACPF plus 970 Diode laser, there was more significant decrease in C content with increase in $\mathrm{Ca}, \mathrm{P}, \mathrm{F}$ wt $\%$ and $\mathrm{Ca} \backslash \mathrm{P}$ ratio compered to CPP-ACPF alone. This reflected an increase in the enamel remineralization and decrease in its solubility as proved by SEM results. This finding was in agreement with (de Sant'Anna et al., 2009) who reported that low level laser irradiation combined with a photoabsorbing cream was able to preserve the calcium and phosphorus content of enamel during demineralizing challenges and increase the inorganic/organic ratio of dental enamel.

Although application of CPP-ACPF with 970nm diode laser in this study produced the highest Ca, P, F, CAIP, ratio among the experimental groups and provided some beneficial remineralizing effects, ANOVA test revealed no significant different in remineralization effect of all experimental groups and the overall benefit was small. This finding is in agreement with (Heravi et al., 2014) who reported that no valuable remineralization effect of infrared laser application on CPP-ACPF treated enamel demineralized lesion. These findings were also in agreement with 
(Fariba, Jaber 2015) who investigated the Changes in bleached enamel surface after Application of Casein Phosphopeptide-Amorphous Calcium Phosphate (CPP-ACP) and diode Laser and reported that the EDAX analysis revealed no any advantageous effect of this laser treatment on enamel surface. In contrast (de Sant'Anna et al., 2009) assured that, there was a significant reduction of the organic matrix and possible modification in the inorganic content in enamel treated with the low-level diode laser and fluorinated photo absorbing cream.

Finally, the discrepancy observed between the outcomes of this study and those of previous studies may be related to the different laser parameters including pulse energy, pulse duration, repetition rate, energy density, and period of irradiation and application of CPP-ACPF, or to the application of water and air cooling during treatment.

This study is a relative study, the actual hardness and enamel thickness was not estimated and so there is a definite need for more laser and CPP-ACPF comparative studies to compare their performance clinically to determine which system can be used for short time and less cost.

\section{Conclusion:-}

1. When demineralized enamel was followed by CPP-ACPF alone better morphological results as well as increased ionic wt\% was observed compared to control group.

2. CPP-ACPF plus (970nm) laser application resulted in more reduction in morphological enamel defects compared to CPP-ACPF group and significant increase in $\mathrm{Ca}, \mathrm{P}, \mathrm{F}$ levels was recorded compared to control group.

\section{References:-}

1. Geraldo-Martins VR., Lepri CP. And Palma-Dibb RG.: Influence of Er, Cr: YSGG laser irradiation on enamel caries prevention. Lasers Med Sci; 28:33-9, 2013.

2. Hemagaran and Neelakantan: Remineralization of tooth structure-The future of dentistry. International Journal of Pharm Tech Research;6(2):487-493, 2013.

3. Patil N., Choudhari S., Kulkarni S. and Joshi SR.: Comparative evaluation of remineralizing potential of three agents on artificially demineralized human enamel: An in vitro study. J Conserv Dent; 16:116-20, 2013.

4. Rose RK.: 34:427-431, 2000. Binding characteristics of Streptococcus mutans for calcium and casein phosphopeptide. Caries Res;

5. Li Y and Hsu CY.: Laser-induced compositional changes on enamel: a FT-Raman study. J Dent; 35:226-30, 2007.

6. De Sant'anna GR, dos Santos EA, Soares LE, do Espirito Santo AM, Martin AA and Duarte DA.: Dental enamel irradiated with infrared diode laser and photoabsorbing cream: Part 1 -- FT-Raman Study. Photomed Laser Surg; 27:499-507, 2009.

7. Moslemi Effects of ER, Cr:YSGG M, Fekrazad R, Tadayon N, Ghorbani M, Torabzadeh H and Shadkar MM.: laser irradiation and fluoride treatment on acid resistance of the enamel. Pediatr Dent; 31:409-13, 2009.

8. Margeas R.: Remineralization with a unique delivery system. Inside Dentistry; p.:863, 2006.

9. Scaramucci T, Carvalho JC, Hara AT and Zero DT.: causes of dental erosion: intrinsic factors. Berlin: Springer International Puplishing; pp. 35-67, 2015.

10. Shahabi M, Moosavi H, Gholami A and Ahrari F.: In vitro effects of several surface preparation methods on shear bond strength of orthodontic brackets to caries-like lesions of enamel. Eur J Pediatr Dent;13:197-202, 2012.

11. Jayarajan J, Janardhanam Pand, Jayakumar P.: Efficacy of CPP-ACP and CPP-ACPF on enamel remineralization- an in vitro study using scanning electron microscope and DIAGNOdent. Indian J Dent Res; 22:77-82, 2011.

12. Ogaard B.: White spot lesions during orthodontic treatment. SeminOrthod; 14(3):183-193, 2008.

13. Bassett DC, Mezaros R, Orzol D.: A new Phtomed Laser surg;29 (7):493-500, 2013.

14. Holler BE, Friedel KH, Jung H, Hiller KA and Schmalz G.: Flouride uptake and distribution in enamel and dentin after application of different fluoride solutions. Clin Oral Investig; 6:137-44, 2002.

15. Samuel, DA and Rubinstein: Microhardness of enamel restored with fluoride and non-flouride dental materials. Braz Dent J.;12:35-38, 2001.

16. Hervi F, Farzaneh A, Mahdavi M and Basafa S.: Comparative evaluation of of the effect of Er:YAG laser and low level laser irradiation combined with CPP-ACPF cream on treatment of enamel caries .J Clin ExpDent; 6(2):e121-e126, 2014. 
17. Ahrari F, Mahdavi M and Basafa S.: Comparative evaluation of the effect of Er:YAG laser on treating enamel caries.J Clin Exp Dent; 8(2): 211-223, 2014.

18. Asl-aminabadi N, Najafpour E, Samiei et al: Laser-casin phosphopeptide effect on remineralization of early enamel lesions in primary teeth: J Clin Exp Dent; 7(2):e261-e267, 2015.

19. Xue Wei, Cong Fu, Keith Savino and Matthew Z.: Carbonated hydroxyapatite coatings aligned crystal domains. Department of chemical engineering and the laboratory for laser energetics, University of Rochester;10:2, 2012. 